\title{
Positron Beam Studies of Radiation Damage Induced by Various Energy Heavy Ions of $\mathrm{Xe}^{26+}$ in Iron
}

\author{
P. HorodeK ${ }^{a, b, *}$, A.G. Kobets ${ }^{b, c}, K_{\text {. SiemeK }}^{a, b}$ And V.A. Skuratov ${ }^{b, d, e}$ \\ ${ }^{a}$ Institute of Nuclear Physics Polish Academy of Sciences, PL-31342 Krakow, Poland \\ ${ }^{b}$ Joint Institute for Nuclear Research, 6 Joliot Curie Str., 141980 Dubna, Russia \\ ${ }^{c}$ Institute of Electrophysics and Radiation Technologies NAS of Ukraine, \\ Chernyshevsky Str. 28, 61002 Kharkov, Ukraine \\ ${ }^{d}$ National Research Nuclear University MEPhI, Kashirskoye sh. 31, 115409 Moscow, Russia \\ ${ }^{e}$ Dubna State University, Universitetskaya 19, 141980 Dubna, Russia
}

\begin{abstract}
Experimental studies of pure iron exposed to $\mathrm{Xe}^{26+}$ irradiation are reported. Implantations were made with the fluence of $5 \times 10^{13}$ ions $/ \mathrm{cm}^{2}$ using $167 \mathrm{MeV}$ ions moderated to $122.5,77.0$, and $44.5 \mathrm{MeV}$. Investigations were performed with variable energy positron beam (VEP). Doppler broadening spectroscopy (DB) was applied. The analysis of obtained results gives information about the presence of various kind of open-volume defects. Two layers with different values of positron diffusion lengths were found in implanted samples. The deeper one was more defected.
\end{abstract}

DOI: 10.12693/APhysPolA.136.318

PACS/topics: 61.80.Jh, 61.82.Bg, 78.70.Bj

\section{Introduction}

Recently the studies of materials exposed to ion irradiation has seemed to be a popular trend of research. The ions contact with the implanted target results in a strong destruction of its structure. In that way a number of lattice defects such as vacancies, their clusters, dislocations, voids etc. are introduced on the path of energetic ions. Their presence has an impact on the material properties. Swelling, hardening, creep, and embrittlement of target appear and cause faster wear [1]. On the other hand, structural defects can be introduced by ions purposely to improve attributes of a given material effectively. The good example for the latter case is production of vacancies by ion implantation before hydrogen insertion. It was experimentally proved that earlier introduced damages can effectively increase the hydrogen absorption rate [2]. For this reason studies of defects induced by ions are provided to develop our knowledge about material sensitivity to various irradiation conditions $[3,4]$.

In this paper the positron beam investigations of pure iron irradiated with different energy heavy ions were reported. Iron was chosen as the object of these tests because of its multidisciplinary character. This is a proper bcc metal for both theoretical and experimental studies. On the other hand, iron is the main component of steel commonly applied in industry and many conclusions based on iron are similar to its alloys.

*corresponding author; e-mail: pawel.horodek@ifj.edu.pl
There are some interesting reports regarding ion implanted iron. For instance, Jenkins et al. [5] in the TEM tests observed a lack of irradiation-induced damages in the case of $40 \mathrm{keV}-240 \mathrm{keV}$ self-implantation. Defects were presented only for heavier ions and the defect yield increased with ion mass. In turn, Drako and Gumanskij [6] found the hexagonal $\varepsilon$-phase in the $\mathrm{N}$ and $\mathrm{C}$ modified layer. Sanghera and Salvani [7] proved that in the case of $\mathrm{N}$ implantation of iron in the low energy range $(2-5 \mathrm{keV})$ nitrogen concentration increases and saturates. This feature depends only on the fluence and not on the current of the beam. The studies carried out by Rutherford and Duffy [8] on the basis of simulations proved that electronic excitation plays an important role in modeling of metals exposed to heavy ion irradiation.

In turn, the results reported in this paper were obtained using the positron annihilation spectroscopy (PAS) method being strongly sensitive to detection of open-volume defects such as vacancies and their clusters. This is a well-known method used in defect studies [911]. Contrary to the conventional PAS tests the research carried out on positron beams can reflect changes close to the surface [12].

The aim of this study is determination of positron annihilation characteristics in the iron samples irradiated with various $\mathrm{Xe}^{26+}$ ions energy using a slow positron beam. We intend to relate the obtained parameters with discussion about the presence of the irradiation-induced defects.

\section{Experimental details}

The samples of $99.99 \%$ purity iron with the dimensions $10 \times 10 \times 1 \mathrm{~mm}^{3}$ were studied. Firstly, they were 
washed in a ultrasound cleaner for $1 \mathrm{~h}$ at $50^{\circ} \mathrm{C}$, then annealed for $4 \mathrm{~h}$ at $1000^{\circ} \mathrm{C}$ under the vacuum conditions of $10^{-5}$ Torr and cooled down in a closed furnace to room temperature.

Irradiation with the $\mathrm{Xe}^{26+}$ ions at the fluence of $5 \times 10^{13}$ was performed at IC-100 cyclotron in the Flerov Laboratory of Nuclear Reaction (FLNR) at Joint Institute for Nuclear Research (JINR) in Dubna. The samples were covered with $\mathrm{Al}$ degraders to moderate energy of the primary beam that was $167 \mathrm{MeV}$. Al foils with the thickness of $3.0,6.5$, and $9.5 \mu \mathrm{m}$ were applied. According to the SRIM calculations [13], the mean energy of ions implanted into iron was 122.5, 77.0, and 45.5 $\mathrm{MeV}$, respectively. The average $\mathrm{Xe}^{26+}$ flux was $5 \times 10^{9} \mathrm{~cm}^{-2} \mathrm{~s}^{-1}$. The temperature during irradiation was not higher than $80^{\circ} \mathrm{C}$. The simulated ion and vacancy distributions are shown in Fig. 1. According to these calculations the implanted range is ca. 5, 6, and $8 \mu \mathrm{m}$ for $45.5,77$, and $122.5 \mathrm{MeV} \mathrm{Xe} \mathrm{X}^{26+}$ ions, respectively.
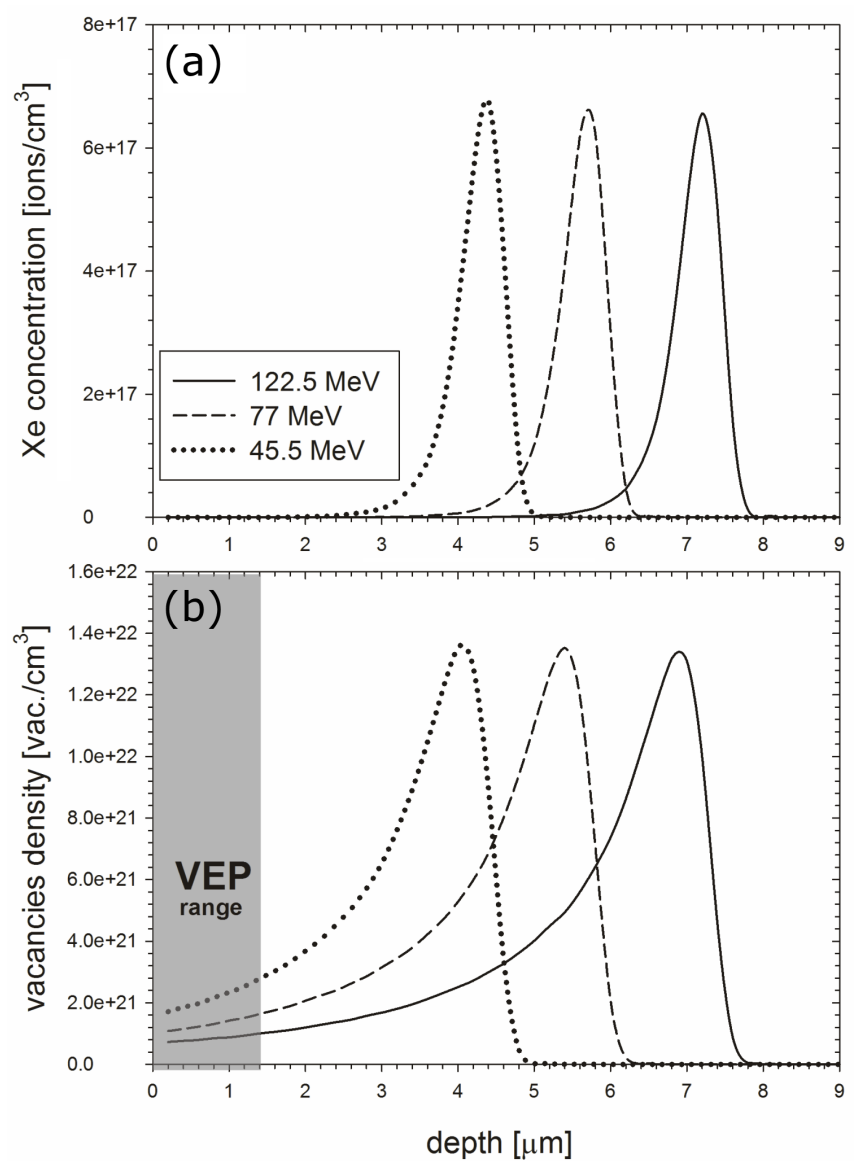

Fig. 1. SRIM calculations of ion (a) and vacancy (b) concentration depth distributions. The marked area in (b) represents the depth scanned by VEP.

The Doppler broadening (DB) spectra of studied samples were measured at room temperature using VEP at JINR [14]. The positrons formed in the $3 \mathrm{~mm}$ diameter flux with the intensity of $10^{6} \mathrm{e}^{+} / \mathrm{s}$ were implanted with incident energy range between $50 \mathrm{eV}$ and $36 \mathrm{keV}$. The annihilation gamma quantum was registered with the HPGe detector with $1.2 \mathrm{keV}$ at $511 \mathrm{keV}$ energy resolution. The registered annihilation line was analysed by extraction of the so-called S parameter. It is given as a ratio of the area below the central part of the annihilation line to the total area under this line. It defines the contribution of annihilating electron-positron pairs with a low momentum. They mostly occur at open volume defects. The energy window for calculation of the $\mathrm{S}$ parameter is $511 \pm 1.1 \mathrm{keV}$. In turn, the W parameter is calculated as the ratio of the area under the wing part of annihilation line to the whole area under the line. The connection of two mentioned parameters gives limited information about a kind of defects.

\section{Results and discussion}

The dependencies of S parameter on the positron implantation energy (bottom axis) and the mean implantation depth (top axis) for the well annealed (defect-free) and irradiated samples are visible in Fig. 2a. The mean implantation depth $(z)$ was estimated using the formula

$$
z=\frac{A}{\rho} E^{n},
$$

where $A=2.62 \mu \mathrm{g} \mathrm{cm}^{-2} \mathrm{keV}^{-n}, n=1.579$, and the density $\rho$ is $7.87 \mathrm{~g} / \mathrm{cm}^{3}$ [15]. In this way the depth of $1.4 \mu \mathrm{m}$ below the surface was investigated.

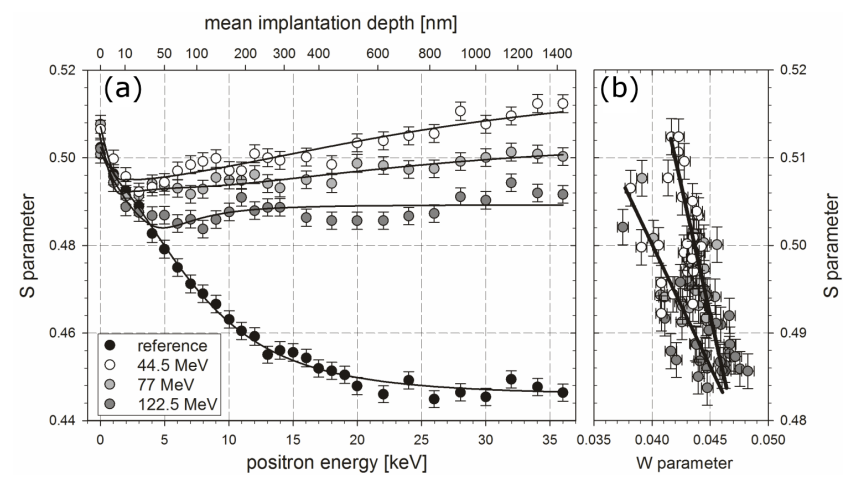

Fig. 2. The measured $\mathrm{S}$ parameter as a function of positron incident energy. The top axis represents the mean positron implantation depth. The solid lines in (a) represent the best fit of diffusion equation to the corresponding experimental points. The dependency $\mathrm{S}$ versus $\mathrm{W}$ is shown in (b) for irradiated samples.

In Fig. 2a the $\mathrm{S}$ parameter decreases with the energy achieving saturation in the case of the reference sample. This is a typical profile for this kind of material [16]. However, profiles from the irradiated specimens decrease also in the range of low positron energies, but do not reflect the stabilization. The second part of profiles for irradiated samples is characterized by a slow $\mathrm{S}$ parameter increase. The greater S parameter value in higher positron energies range for different samples proves the existence 
of irradiation induced defects. Their concentration is higher for the samples implanted with lower energy of $\mathrm{Xe}^{26+}$. In our previous VEP studies with $167 \mathrm{MeV} \mathrm{Xe}^{26+}[17-19]$ and $107 \mathrm{MeV} \mathrm{Kr}^{17+}[20]$ ions obtained profiles were characterized only by the decrease and saturation of $\mathrm{S}$ parameter with the positron energy. Different behavior of the reported distributions for the implanted targets can be explained by reaching the implantation range marked by the Bragg peak. This can be the reason for the $\mathrm{S}$ parameter increase in the second part of profiles.

In Fig. 2b the dependency of $S-W$ is shown. All experimental points are fitted by two straight lines with different slopes. This feature is an evidence of various type of defects present in the studied depth. Using the VEPFIT code [21] for fitting the model function being the positron diffusion equation to the obtained profiles makes a broader analysis possible. In the case of irradiated samples two layers were approached in the fitting procedure. The best fits are marked with the solid black lines in Fig. 2a and the results are plotted in Fig. 3.

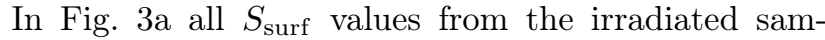
ples (white circles) are similar showing that no important changes were introduced by irradiation in the surfaces. $S_{1}, S_{2}$ (grey triangles) present the $\mathrm{S}$ values at the end of
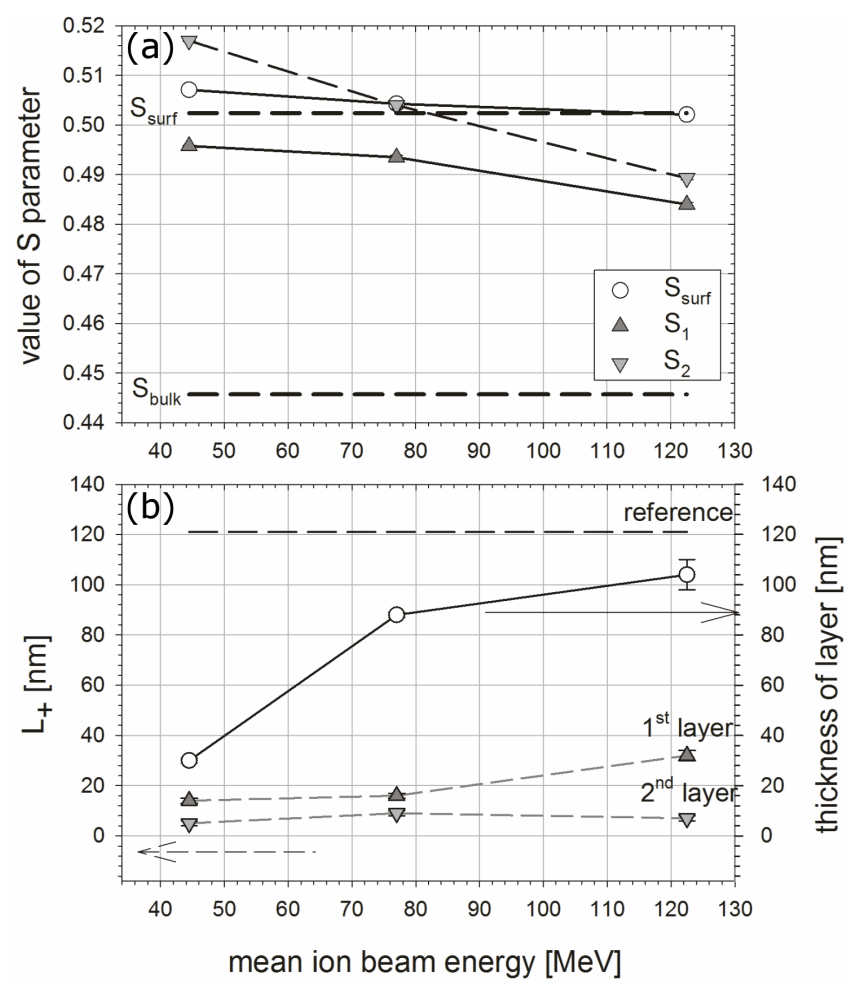

Fig. 3. (a) Values of $S_{\text {surf }}, S_{1}$ and $S_{2}$ parameters and (b) positron diffusion length and thickness of the first layer obtained from VEPFIT in dependence on the ion beam energy. The dashed lines marked with $S_{b u l k}$ and $S_{\text {surf }}$ represent values of $S$ parameters for saturation and surface of reference sample. detected layer. They are much higher for irradiated specimens in comparison to the reference one $\left(S_{\text {bulk }}\right.$ marked with dashed line). The presence of defects induced by the irradiation along the initial part of the implantation path is confirmed. Additionally, the $\mathrm{S}$ values are higher in the second layer pointing out that this is more defected in the depth range studied by VEP.

In Fig. 3b the thicknesses of the first layer (white circles) and positron diffusion lengths in both layers (grey triangles) depending on ion energy are shown. The dashed line represents the value of $L_{+}$for the nonirradiated sample [19]. The obtained value is in good agreement with that for the defect-free iron. Irradiation with lower energy reduces the layer and also causes shortening of $L_{+}$indicating the production of open-volume defects. The positron diffusion length is connected with the defect concentration $C_{v}$ by the following relation

$$
C_{v}=\frac{1}{\tau_{\text {bulk }} \mu}\left[\left(\frac{L_{\mathrm{bulk}}}{L_{+}}\right)^{2}-1\right],
$$

where $\mu$ is the trapping coefficient, $\tau_{\text {bulk }}$ and $L_{\text {bulk }}$ are the mean positron lifetime and diffusion length in a non-defected structure, respectively. $L_{+}$represents the positron diffusion length in the irradiated sample. According to this equation defect concentration rises when $L_{+}$decreases. In our case shortening of $L_{+}$for the irradiated samples is observed. Shorter $L_{+}$in the $2^{\text {nd }}$ layer points out to a higher defect concentration compared to the $1^{\text {st }}$ one.

\section{Summary}

$\mathrm{Xe}^{26+}$ implantation was performed into iron with different mean energies. The presence of irradiation induced defects is confirmed in all VEP characteristics. Two defected layers are recognized in the measured profiles. The thickness of the $1^{\text {st }}$ layer increases with increase in ion energy. In the investigated depth defects concentration is the highest in the sample irradiated with the lowest energy. Probably the different kind of defects were generated in all specimens.

\section{References}

[1] G.S. Was, Fundamentals of Radiation Materials Science: Metals and Alloys, 2nd ed., Springer, Berlin, 2016.

[2] H. Abe, H. Uchida, Y. Azum, A. Uedono, Z.Q. Chen, H. Itoh, Nucl. Inst. Meth. B 206, 224 (2003).

[3] A. Olejniczak, V.A. Skuratov, Nucl. Inst. Meth. B 326, 33 (2014).

[4] M. Kulik, D. Kołodyńska, A. Bayramov, A. Drozdziel, A. Olejniczak, J. Żuk, Spectrochim. Acta A 198, 222 (2018).

[5] M.L. Jenkins, C.A. English, B.L. Eyre, Philos. Mag. A 38, 97 (1978).

[6] V.M. Drako, G.A. Gumanskij, Radiat. Eff. 66, 101 (1982). 
[7] H.K. Sanghera, J.L. Sullivan, Nucl. Instrum. Meth. Phys. Res. B 152, 65 (1999).

[8] A.M. Rutherford, D.M. Duffy, Nucl. Instrum. Meth. Phys. Res. B 267, 53 (2009).

[9] J. Dryzek, E. Dryzek, B. Cleff, Appl. Surf. Sci. 116, 236 (1997).

[10] E. Dryzek, J. Kuriplach, J. Dryzek, J. Phys. Condens. Matter 10, 6573 (1998).

[11] E. Dryzek, M. Sarnek, K. Siemek, Nukleonika 58, 215 (2013).

[12] F. Tuomisto, I. Makkonen Rev. Mod. Phys. 85, 1583 (2013).

[13] J.F. Ziegler, Ion Implantation Science and Technology, Academic Press, New York, 1988.

[14] P. Horodek, A.G. Kobets, I.N. Meshkov, A.A. Sidorin, O.S. Orlov, Nukleonika 60, 725 (2015).
[15] J. Dryzek, P. Horodek, Nucl. Instrum. Methods B 266, 4000 (2008).

[16] J. Dryzek, E. Dryzek, R. Krause-Rehberg, F. Reddmann, Tribol. Lett. 11, 121 (2001).

[17] P. Horodek, J. Dryzek, V.A. Skuratov, Vacuum 138, 15 (2017).

[18] J. Dryzek, P. Horodek, V.A. Skuratov, Acta Polon. A 132, 1585 (2017).

[19] P. Horodek, J. Dryzek, V.A. Skuratov, Rad. Phys. Chem. 122, 60 (2016).

[20] P. Horodek, V.A. Skuratov, Surf. Coat. Technol. 296, 65 (2016).

[21] A.V. Veen, H. Schut, M. Clement, A. Kruseman, M.R. Ijpma, J.M.M. De Nijs, Appl. Surf. Sci. 85, 216 (1995). 